\title{
EDITORIAL \\ Modern trends in applied probability closing a special issue of overview papers
}

\author{
Ross McVinish ${ }^{1} \cdot$ Yoni Nazarathy ${ }^{1}$. Giang Nguyen ${ }^{2}$
}

Received: 24 December 2021 / Revised: 27 December 2021 / Accepted: 28 December 2021 /

Published online: 28 January 2022

(c) The Author(s), under exclusive licence to Springer Science+Business Media, LLC, part of Springer Nature 2022

The special issue 'Modern Trends in Applied Probability', which was spread over multiple volumes, concludes in this volume with a paper by Yiqiang Q. Zhao. The special issue arose out of the 20th INFORMS-APS conference held in Brisbane, Australia in July 2019. It is comprised of overview papers surveying multiple aspects of queueing theory and applied probability. Following the conference, we initiated a process which involved submission of an expression of interest and open peer review between overview paper authors, as well as blind external referees. A total of eight expressions of interests were submitted and now at the closing of this special issue, five out of the eight proposals matured into extensive overview papers and made their way to publication.

The four papers that have been published previously for the special issue are: 'Product forms for FCFS queueing models with arbitrary server-job compatibilities: an overview' by Kristen Gardner and Rhonda Righter, 'A survey on skill-based routing with applications to service operations management' by Jinsheng Chen, Jing Dong, and Pengyi Shi, 'A survey of parameter and state estimation in queues' by Azam Asanjarani, Yoni Nazarathy, and Peter Taylor, and 'A survey of queueing systems with strategic timing of arrivals' by Moshe Haviv and Liron Ravner. The first two survey papers appeared in Sept-Oct 2020 (Vol. 96, Nos 1-2). The third paper appeared in Jan-Feb 2021 (Vol. 97, Nos 1-2), and the fourth paper appeared in Sept-Oct 2021 (Vol. 99, Nos 1-2).

We are now happy to conclude the special issue with the fifth paper by Yiqiang Q. Zhao. This paper deals with a method for analysing tail asymptotics of twodimensional queueing systems: the kernel method. The paper provides an extensive literature survey of tail asymptotics of the stationary distribution of such models together with a detailed description of how the kernel method is applied to such systems.

\footnotetext{
Yoni Nazarathy y.nazarathy@uq.edu.au

1 The University of Queensland, Brisbane, Australia

2 The University of Adelaide, Adelaide, Australia
} 
Together, the breadth and depth of these papers is impressive as they illustrate the rich nature of queueing theory and applied probability. The areas surveyed in these papers use techniques of Markov chains, game theory, diffusion processes, statistics, complex analysis, and other tools to deal with interesting operational problems and mathematical challenges. Each overview paper includes an extensive survey of the relevant literature, with the total number of references of papers in this special issue at over 400. We hope you find these overview papers useful and insightful.

It was a pleasure to accompany the eleven authors of these five papers on their journey of preparation of these surveys. As always, we are also grateful to anonymous referees whose input was crucial. As for the follow-up conference to the 20th INFORMS-APS, it was first scheduled to run in 2021 and has since been twice rescheduled due to hardships of the COVID pandemic. It is now scheduled to run in Nancy, France in July 2023, and we hope that by then many members of the research community will be able to meet in person.

Guest Editors: Ross McVinish, Yoni Nazarathy, and Giang Nguyen.

Publisher's Note Springer Nature remains neutral with regard to jurisdictional claims in published maps and institutional affiliations. 